\title{
GMR
}

\section{Development of a transposon-based marker system for mutation breeding in sorghum (Sorghum bicolor L.)}

\author{
S.B. Im ${ }^{1 *}$, S.-J. Kwon ${ }^{1,2 *}$, J. Ryu ${ }^{1}$, S.W. Jeong ${ }^{1}$, J.B. Kim ${ }^{1}$, J.-W. Ahn ${ }^{1,2}$, \\ S.H. Kim ${ }^{1}$, Y.D. Jo ${ }^{1}$, H.-I. Choi ${ }^{1}$ and S.-Y. Kang ${ }^{1}$ \\ ${ }^{1}$ Advanced Radiation Technology Institute, \\ Korea Atomic Energy Research Institute, Jeongeup, Korea \\ ${ }^{2}$ Radiation Biotechnology and Applied Radioisotope Science, \\ University of Science and Technology, Daejeon, Korea \\ *These authors contributed equally to this study. \\ Corresponding authors: S.-J. Kwon / S.-Y. Kang \\ E-mail: soonjaekwon@kaeri.re.kr/sykang@kaeri.re.kr
}

Genet. Mol. Res. 15 (3): gmr.15038713

Received April 18, 2016

Accepted June 6, 2016

Published September 16, 2016

DOI http://dx.doi.org/10.4238/gmr.15038713

Copyright (C) 2016 The Authors. This is an open-access article distributed under the terms of the Creative Commons Attribution ShareAlike (CC BY-SA) 4.0 License.

\begin{abstract}
Under certain circumstances, transposable elements (TE) can create or reverse mutations and alter the genome size of a cell. Sorghum (Sorghum bicolor L.) is promising for plant transposon tagging due to its small genome size and its low content of repetitive DNA. We developed a marker system based on targeted region amplification polymorphisms (TE-TRAP) that uses the terminal inverted repeats (TIRs) of transposons. A total of 3816 class 2 transposons belonging to the PIF/Harbinger family were identified from the whole sorghum genome that produced five primers, including eight types of TIRs. To define the applicability and utilization of TE-TRAP, we used 21 individuals that had been bred after $\gamma$-ray irradiation. In total, 31 TE-
\end{abstract}


TRAP, 16 TD, and 21 AFLP primer combinations generated 1133, 223, and 555 amplicons, respectively. The percent polymorphic marker was $62.8,51.1$, and $59.3 \%$ for the TE-TRAP, TD, and AFLP markers, respectively. Phylogenetic and principal component analyses revealed that TE-TRAP divided the 21 individuals into three groups. Analysis of molecular variance suggested that TE-TRAP had a higher level of genetic diversity than the other two marker systems. After verifying the efficiency of TE-TRAP, 189 sorghum individuals were used to investigate the associations between the markers and the $\gamma$-ray doses. Two significant associations were found among the polymorphic markers. This TE-based method provides a useful marker resource for mutation breeding research.

Key words: Sorghum; Transposable element; Mutation breeding; $\gamma$-ray; TE-TRAP

\section{INTRODUCTION}

Sorghum (Sorghum bicolor L.) is a C4 monocot species and one of the most important cereal crops. As a domesticated crop plant, it is used not only for human food, but also for fodder, animal feed, bio-energy, and as building material (Doggett, 1988; Rooney and Waniska, 2000; Lombardi et al., 2015). The complete genome sequence of sorghum was released for public use in early 2008, which enabled researchers to understand its complex traits at the DNA sequence level (Paterson et al., 2009). Sorghum has great potential for use in plant genomics due to its adaptation to harsh environments, the availability of a diverse germplasm collection, its small genome size, and the co-linearity of its genome with other cereal genomes (Bennetzen et al., 1998).

Mutation breeding is a useful method for crop improvement and it has played a critical role in sustainable agriculture. Mutation breeding using $\gamma$-rays directly produces mutant varieties without having to go through the otherwise lengthy and laborious process of conventional breeding. Irradiation has been successfully used for mutation breeding in various crops and ornamental plants and has proven to be a promising means of producing new genetic variants (Hara et al., 2003; Song and Kang, 2003).

Transposable elements (TEs) were first discovered by Barbara McClintock nearly 60 years ago using classical genetics in maize (McClintock, 1950). TEs are ubiquitous components of almost every investigated eukaryotic genome and repetitive sequences can change their location within a genome. TEs contribute significantly to the size, structure, and plasticity of genomes and also play an active role in genome evolution by helping their hosts adapt to new conditions by conferring useful characteristics (Zhang and Wessler, 2004; Wessler, 2006). TEs are categorized into two classes: class 1 elements, or retrotransposons, which move using a copy-and-paste mechanism to amplify intermediate RNA and class 2 elements, or DNA transposons, which move within the genome by excising from their original location to a new region using a cut-and-paste mechanism (Feschotte et al., 2002). Class 2 TEs are categorized into several subfamilies (Kunze et al., 1997). Among these, miniature inverted-repeat transposable elements (MITEs) are characterized by their small size (usually less than $500 \mathrm{bp}$ ), their lack of coding capacity, and their tendency to have short terminal

Genetics and Molecular Research 15 (3): gmr.15038713 
inverted repeats (TIRs) (Feschotte et al., 2002). MITEs are often discovered close to or within genes where they may affect gene expression (Santiago et al., 2002). MITEs can be further categorized into superfamilies, which include five discovered and characterized superfamilies among plant genomes (Feschotte and Pritham, 2007).

Among polymerase chain reaction (PCR)-based DNA marker systems, amplified fragment length polymorphism (AFLP), transposon display (TD), and targeted region amplification polymorphism (TRAP) are commonly and extensively used tools for assessment of variability in crops and genetic resources. AFLP analysis is a DNA fingerprinting technique developed by Vos et al. (1995). AFLP markers are dominant markers that detect high amounts of polymorphism (Mueller and Wolfenbarger, 1999). TD is a modified version of the conventional AFLP technique that has been applied successfully to a conserved sequence motif of a MITE element in maize (Casa et al., 2000). In this study, we developed a TE-TRAP marker system as a modification of the conventional TRAP marker system. The TE-TRAP marker system was developed to select a group of initial mutant plants after $\gamma$-ray irradiation. TE-TRAP is a simple PCR-based marker technique, which uses fixed primers from class 2 MITE TE superfamily sequence information and arbitrary primers that target intron or exon regions with an AT- or GC-rich core to amplify DNA fragments.

Previously, miniature Ping sequences in rice, which contain a putative $\mathrm{P}$ instability factor (PIF) family, were activated using $\gamma$-rays (Kikuchi et al., 2003). TE activity can be induced by environmental factors and, in particular, by physical stressors, such as $\gamma$-rays. For this reason, we conducted $\gamma$-ray irradiation of sorghum.

The objective of our study was to evaluate three different marker systems (TE-TRAP, TD, and AFLP) for genetic diversity, polymorphisms, and genetic distance, and to confirm the applicability and value of the TE-TRAP marker system developed in this study. We conducted an experiment to investigate the association between markers and $\gamma$-ray doses and to show that the TE-TRAP marker system is suitable for mutation selection.

\section{MATERIAL AND METHODS}

\section{Plant materials and DNA extraction}

Nine sorghum cultivars from Korea, the United States, and South Africa (Table 1) were used in this study. The 1000 dry seeds of each cultivar were irradiated by four different dose $(100,200,300,400 \mathrm{~Gy}) \gamma$-rays emitted from a $\left[{ }^{60} \mathrm{Co}\right]$ source at the Korea Atomic Energy Research Institute (KAERI). The seeds were planted in plots $(3 \times 6 \mathrm{~m})$ and row spacing of 20 and $60 \mathrm{~cm}$, respectively. After a month of germination, fresh leaf materials were harvested from five individuals each treated dose and original cultivars.

The genomic DNA was extracted from leaves of $\mathrm{M}_{1}$ plant and each control using a modified cetyl trimethylammonium bromide method protocol (Doyle and Doyle, 1987). Genomic DNA was quantified using a NanoDrop ND-1000 spectrophotometer instrument (Thermo Fisher Scientific, Waltham, MA, USA) and it was normalized to a uniform concentration $(100 \mathrm{ng} / \mu \mathrm{L})$.

\section{Sequence analysis and primer design}

Whole sorghum genome sequences (Plant MITE databases; http://pmite.hzau.edu.cn)

Genetics and Molecular Research 15 (3): gmr.15038713 
was employed to identify class 2 MITEs using MITE Digger program (Yang, 2013). The confirmed MITEs were compared with the P-MITE database (http://pmite.hzau.edu.cn) for classification into TE super families. The compositions of five categories of MITEs (CACTA, hAT, PIF/Harbinger, Tc1/Mariner, Mutator) are shown in Figure 1. Using the PIF/Harbinger element sequence, eight types of TIRs were discovered and were aligned and matched in pairs with degenerate sequences. Sb_PIF primers were designed to match a 19-20 bp size corresponding to the 20-bp TIR of the PIF/Harbinger element. A Zea mays (Zm) primer, Zm PIF_1, was designed using the TIR sequences of Z. mays mPIF deposited in the GenBank database (accession No. AF416298-AF416329). Isaac maps were designed from consensus sequences obtained from the GenBank database using basic information obtained from Lee et al. (2005).

Table 1. Cultivar name and origin of Sorghum germplasm used in this study.

\begin{tabular}{l|l|l}
\hline No. & Cultivar name & Origin \\
\hline 1 & DINE-A-MITE & Unknown \\
\hline 2 & IS645 & United States \\
\hline 3 & IS2868 & South Africa \\
\hline 4 & Moktak & Korea (breeding cultivar) \\
\hline 5 & Banwoldang & Korea (breeding cultivar) \\
\hline 6 & Chal II & Korea (native species) \\
\hline 7 & KLSO79125 & Korea (native species) \\
\hline 9 & KLSO79075 & Korea (native species) \\
\hline
\end{tabular}

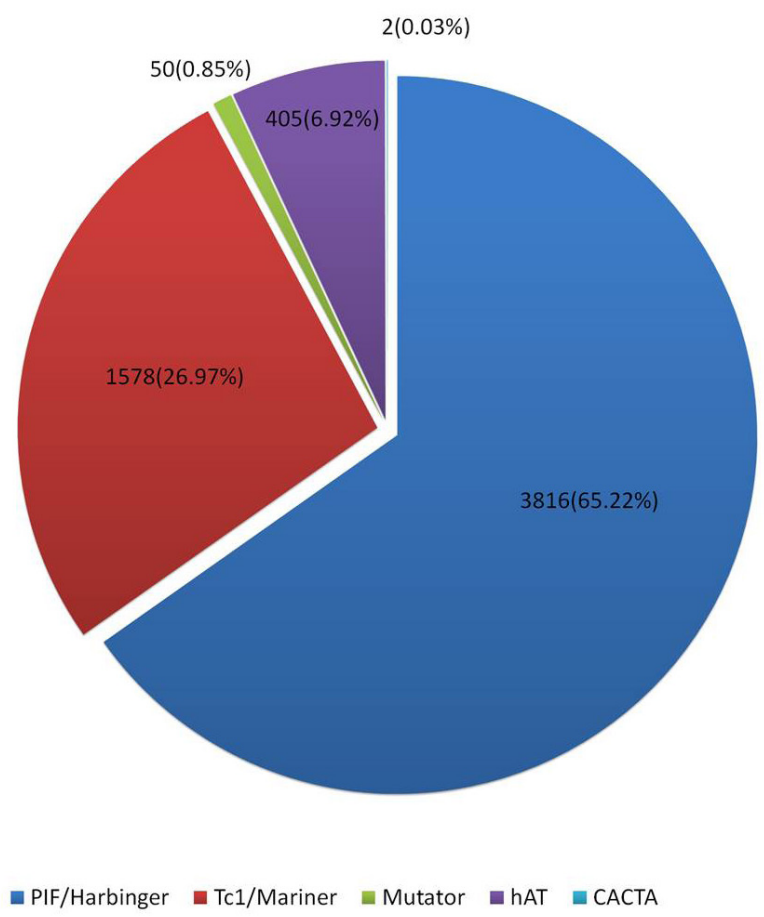

Figure 1. Distribution of class 2 transposable elements in the sorghum (Sorghum bicolor) whole genome sequence. 


\section{Generation of markers}

The TE-TRAP, TD, and AFLP marker systems were applied to 21 individuals (five individuals each treated dose and a control) of the sorghum variety IS2868, which were irradiated with five $r$-ray doses $(0,100,200,300$, and 400 Gy). TE-TRAP amplification was carried out using a modified version of the protocol from Hu et al. (2005), which included seven fixed and six arbitrary primers. Arbitrary primers were used to target intron or exon regions with an AT- or GC-rich core to amplify the DNA fragments (Li and Quiros, 2001). The arbitrary primers used in this study were based on those described by Hu et al. (2005). A total volume of $20 \mu \mathrm{L}$ was used for PCR amplification, which contained $2 \mu \mathrm{L}$ genomic DNA (10 $\mathrm{ng} / \mu \mathrm{L}), 1 \mu \mathrm{L}$ fixed $(10 \mathrm{pmol} / \mu \mathrm{L})$ and arbitrary primer $(2 \mathrm{pmol} / \mu \mathrm{L}), 0.4 \mu \mathrm{L} d \mathrm{NTPs}(10 \mathrm{mM})$,

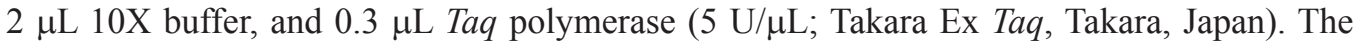
PCR amplification was performed by initially denaturing template DNA at $94^{\circ} \mathrm{C}$ for $2 \mathrm{~min}$, followed by 5 cycles at $94^{\circ} \mathrm{C}$ for $45 \mathrm{~s}, 35^{\circ} \mathrm{C}$ for $45 \mathrm{~s}$, and $72^{\circ} \mathrm{C}$ for $90 \mathrm{~s}$, then 35 cycles at $94^{\circ} \mathrm{C}$ for $45 \mathrm{~s}, 50^{\circ} \mathrm{C}$ for $45 \mathrm{~s}$, and $72^{\circ} \mathrm{C}$ for $90 \mathrm{~s}$, and a final extension at $72^{\circ} \mathrm{C}$ for 7 min to terminate the reaction.

The TD and AFLP marker systems are similar. AFLP was conducted using two endonucleases (MseI and EcoRI), whereas only one endonuclease (MseI) was used for TD. The DNA was fully digested with the endonuclease at $37^{\circ} \mathrm{C}$ overnight and the reaction was terminated at $70^{\circ} \mathrm{C}$ for $2 \mathrm{~min}$. The DNA fragments were ligated to adapters by adding $1 \mu \mathrm{L} \mathrm{T} 4$ DNA ligase to a total volume of $50 \mu \mathrm{L}$ at $20^{\circ} \mathrm{C}$ for $2 \mathrm{~h}$. Ligated DNA was diluted 10 -fold and used for pre-amplification. Pre-amplification was done with the KRMP-0 and the Isaac map primer. PCR performed with one cycle at $72^{\circ} \mathrm{C}$ for $2 \mathrm{~min}$ and at $94^{\circ} \mathrm{C}$ for $3 \mathrm{~min} ; 25$ cycles were run at $94^{\circ} \mathrm{C}$ for $30 \mathrm{~s}, 56^{\circ} \mathrm{C}$ for $30 \mathrm{~s}$, and $72^{\circ} \mathrm{C}$ for $1 \mathrm{~min}$; a final extension was run at $72^{\circ} \mathrm{C}$ for $5 \mathrm{~min}$ to complete the reaction. For selective amplification, the pre-amplified products were diluted 50-fold. The selective amplification consisted of 1 cycle at $94^{\circ} \mathrm{C}$ for $5 \mathrm{~min} ; 10$ "touchdown" cycles of $94^{\circ} \mathrm{C}$ for $30 \mathrm{~s}, 64^{\circ} \mathrm{C}$ for $30 \mathrm{~s}$, and $72^{\circ} \mathrm{C}$ for 1 min with a decrease in annealing temperature by $1^{\circ} \mathrm{C}$ each cycle; 28 cycles at $94^{\circ} \mathrm{C}$ for $30 \mathrm{~s}, 56^{\circ} \mathrm{C}$ for $30 \mathrm{~s}$, and $72^{\circ} \mathrm{C}$ for $1 \mathrm{~min}$; and $72^{\circ} \mathrm{C}$ for $5 \mathrm{~min}$ to terminate the reaction. Primer sequence information of each marker system is shown in Table 2. The amplified products were separated using a caliper LabChip GXII instrument (Caliper Life Sciences, USA) and the collected images were scored manually.

\section{Data analysis}

Each amplified fragment was scored with a binary code ( 1 or 0 for presence or absence, respectively). Based on the 0/1-matrix, we calculated gene diversity (GD), the percentage of monomorphic and polymorphic markers, polymorphic information content (PIC) (Nei, 1972; $\mathrm{Yu}$ et al., 2003), and genetic distance, using the genetic analysis package Power Marker (Liu and Muse, 2005). In addition, genetic differentiation was tested using the $F_{\mathrm{ST}}$ statistic, which estimates pairwise comparisons.

To compare the genetic differentiation of the three marker systems, we used a phylogenetic tree and principal component analysis (PCA). The phylogenetic tree was constructed using the unweighted pair group method with arithmetic mean (Sneath and Sokal, 1973), using the MEGA 6.06 program (Tamura et al., 2007), which is embedded in Power Marker. The PCA was used to classify and discriminate $\gamma$-ray irradiated sorghum individuals,

Genetics and Molecular Research 15 (3): gmr.15038713 
using the XLSTAT software (Addinsoft, 2008, USA). We then conducted analysis of molecular variance (AMOVA) (Schneider et al., 2000) using GenALEx v. 6.501 (Peakall and Smouse, 2006), to support the genetic differentiation information (obtained in the phylogenetic tree and PCA). The AMOVA estimates and separates total molecular variance among and within populations and then examines the significance of separated variance components using permutational testing procedures.

Table 2. Primer names and sequences for each of the three marker systems (TE-TRAP, AFLP, TD) used in the current study.

\begin{tabular}{|c|c|c|c|}
\hline \multicolumn{4}{|l|}{ TE-TRAP } \\
\hline Fixed primer & Sequence $\left(5^{\prime}-3^{\prime}\right)^{1}$ & Arbitrary primer & Sequence $\left(5^{\prime}-3^{\prime}\right)$ \\
\hline Sb_PIF_1a & KYY TGC ATT GTG AGT GCC CT & odd 15 & GCG AGG ATG CTA CTG GTT \\
\hline Sb_PIF_1b & YYT GCA TTG TGA GTG CCC T & odd 26 & CTA TCT CTC GGG ACC AAA C \\
\hline Sb PIF 2 & MYA TGC ATT GAG ACT GGC CT & sa4 & TTA CCT TGG TCA TAC AAC ATT \\
\hline Sb PIF 3 & ACW ACA TCC AAA CAA GGC CT & sa12 & TTC TAG GTA ATC CAA CAA CA \\
\hline Sb_PIF_4 & TTS SGA ACT AAA CAA GGC CT & ga3 & TCA TCT CAA ACC ATC TAC AC \\
\hline $\mathrm{Zm} \_\mathrm{PIF} \_1$ & RVK AAA CAA AYG GGV YC & ga5 & GGA ACC AAA CAC ATG AAG A \\
\hline Isaac Map & ATA GGG TGC GAT TCC GGT AGT G & & \\
\hline \multicolumn{4}{|c|}{ 年 } \\
\hline Adaptor & Sequence (5'-3') & Selective primer & Sequence (5'-3') \\
\hline$E c o$ RI/F & CTC GTA GAC TGC GTA CC & $\mathrm{E} / \mathrm{AA}$ & GAC TGC GTA CCA ATT CAA \\
\hline EcoRI/R & AAT TGG TAC GCA GTC & $\mathrm{E} / \mathrm{TG}$ & GAC TGC GTA CCA ATT CTG \\
\hline$M s e \mathrm{I} / \mathrm{F}$ & GAC GAT GAG TCC TGA G & E/TT & GAC TGC GTA CCA ATT CTT \\
\hline $\mathrm{Msel} / \mathrm{R}$ & TAC TCA GGA CTC AT & M/CAA & GAT GAG TCC TGA GTA ACAA \\
\hline Pre-selective primer & Sequence (5'-3') & $\mathrm{M} / \mathrm{CAC}$ & GAT GAG TCC TGA GTA ACA C \\
\hline EcoRI & GAC TGC GTA CCA ATT C & $\mathrm{M} / \mathrm{CAG}$ & GAT GAG TCC TGA GTA ACA G \\
\hline \multirow[t]{5}{*}{ MseI } & GAT GAG TCC TGA GTAA & $\mathrm{M} / \mathrm{CAT}$ & GAT GAG TCC TGA GTAACA T \\
\hline & & $\mathrm{M} / \mathrm{CTA}$ & GAT GAG TCC TGA GTA ACT A \\
\hline & & $\mathrm{M} / \mathrm{CTC}$ & GAT GAG TCC TGA GTA ACT C \\
\hline & & $\mathrm{M} / \mathrm{CTG}$ & GAT GAG TCC TGA GTA ACT G \\
\hline & & $\mathrm{M} / \mathrm{CTT}$ & GAT GAG TCC TGA GTA ACT T \\
\hline \multicolumn{4}{|l|}{ TD } \\
\hline Adaptor & Sequence (5'-3') & MseI anchor & Sequence $\left(5^{\prime}-3^{\prime}\right)$ \\
\hline KRMA-1 & GAC GAT GAG TCC TGA G & KRMP-0 & GAC GAT GAG TCC TGA GTAA \\
\hline KRMA-2 & TAC TCA GGA CTC AT & KRMP-GA & GAC GAT GAG TCC TGA GTA AGA \\
\hline Selective primer & Sequence $\left(5^{\prime}-3^{\prime}\right)$ & KRMP-GAA & GAC GAT GAG TCC TGA GTA AGAA \\
\hline Sb_PIF_1a & KYY TGC ATT GTG AGT GCC CT & KRMP-GAC & GAC GAT GAG TCC TGA GTA AGA C \\
\hline $\mathrm{Sb}$ PIF $1 \mathrm{~b}$ & YYT GCA TTG TGA GTG CCC T & KRMP-GAG & GAC GAT GAG TCC TGA GTA AGA G \\
\hline Sb_PIF_2 & MYA TGC ATT GAG ACT GGC CT & KRMP-GAT & GAC GAT GAG TCC TGA GTA AGA T \\
\hline Sb_PIF_3 & ACW ACA TCC AAA CAA GGC CT & KRMP-CAC & GAC GAT GAG TCC TGA GTA ACAC \\
\hline Sb PIF 4 & TTS SGA ACT AAA CAA GGC CT & KRMP-CAG & GAC GAT GAG TCC TGA GTA ACA G \\
\hline $\mathrm{Zm}$ PIF_1 & RVK AAA CAA AYG GGV YC & KRMP-CAT & GAC GAT GAG TCC TGA GTA ACA T \\
\hline \multirow{2}{*}{ Isaac map } & ATA GGG TGC GAT TCC GGT AGT G & KRMP-CGT & GAC GAT GAG TCC TGA GTA ACC T \\
\hline & & KRMP-CTA & GAC GAT GAG TCC TGA GTA ACT A \\
\hline
\end{tabular}

The TE-TRAP marker system was used to analyze the association mapping between the markers and 189 individuals (nine accessions with five individuals of each $\gamma$-ray dose and each original cultivars), which were bred after $\gamma$-ray-irradiation, using the TASSEL 5.2.9 software (Bradbury et al., 2007). The associations between the markers and the $\gamma$-ray doses were tested using general linear model (GLM) and mixed linear model (MLM) with K-matrix $(\mathrm{MLM}+\mathrm{K})$ methods.

\section{RESULTS}

\section{Identification of class 2 TEs and primer design}

All five MITE superfamilies belonging to the class 2 TEs were identified in sorghum whole genome sequences (Figure 1). PIF/Harbinger elements were the most numerous 
elements $(65.20 \%)$ and TC1/Mariner elements were the second most numerous $(27.00 \%)$. The number of hAT, Mutator, and CACTA elements was 6.90\%, $50(0.87 \%)$, and $2(0.03 \%)$, respectively. To enrich the amplicons containing the flanking sequences of the PIF/Harbinger transposon, we designed Sb_PIF primer sequences using the outermost 20-bp TIR sequences of the PIF/Harbinger elements.

\section{Analysis of genetic diversity}

TE-TRAP was carried out with 31 primer combinations and a total of 1133 fragments were amplified. These fragments contained 20-50 amplicons ranging in size from 100 to 1500 bp per primer combination. Among these fragments, 421 (37.2\%) were monomorphic and $712(62.8 \%)$ were polymorphic (Table 3$)$. For each primer combination, an average of 37 fragments was scored, and 23 were polymorphic. The highest level of polymorphism (85.4\%) was obtained from primer combination Sb_PIF_3 + Sa12, whereas the lowest (43.2\%) was obtained from primer combination $\mathrm{Zm} \_\mathrm{PIF} 11+\mathrm{Sa} 12$ (Table 4). Genetic diversity and PIC values ranged from 0.124 (Isaac map + sa12) to $0.335($ Sb_PIF_1a + sa4) and from 0.102 (Isaac map + sa12) to 0.264 (Sb_PIF_1a + sa4), with averages of $0 . \overline{2} 14$ and 0.172 , respectively.

\begin{tabular}{l} 
Table 3. Gene diversity and polymorphic information content (PIC) of each marker system (TE-TRAP, TD, \\
and AFLP). \\
\hline \\
\hline Number of markers
\end{tabular}

We carried out the TD amplification with 16 primer combinations that resulted in a total of 223 amplicons. The number of amplicons amplified by each primer combination ranged from six (Sb_PIF_1b + TGC) to 26 (Sb_PIF 3 + GAT) and the sizes of the amplified fragments ranged from $1 \overline{00}$ to $1500 \mathrm{bp}$. A total of $22 \overline{3}$ fragments were scored, 109 (48.9\%) of these were monomorphic, whereas 114 (51.1\%) were polymorphic (Table 3$)$. For each primer combination, on an average, 14 fragments were scored and seven were polymorphic. The highest level of polymorphism $(68.8 \%)$ was obtained with primer combination Sb_PIF_2 + GAC, and the lowest level (33.3\%) was obtained with primer combination Sb_PIF_1 $1 \mathrm{~b}+\overline{\mathrm{T}} \mathrm{GC}$ and Isaac map + GAA (Table 4). The GD and PIC values ranged from 0.054 (Isaac map + GA) to 0.224 (Sb_PIF_3 + GA) and from 0.050 (Isaac map + GA) to 0.179 (Sb_PIF_3 + GA), averaging 0.148 and 0.122 , respectively.

Amplification of $\gamma$-ray-irradiated sorghum sequences using the AFLP marker system generated multiple amplicons and abundant polymorphic fragments. The amplified fragments ranged from 100 to $1500 \mathrm{bp}$ and the number of scorable fragments amplified by the 21 primer combinations ranged from $18(\mathrm{TG}+\mathrm{CTC})$ to $43(\mathrm{TT}+\mathrm{CAT})$. A total of $555 \mathrm{amplicons}$ were scored of which 329 fragments (59.3\%) were polymorphic and 226 fragments $(40.7 \%)$ were monomorphic (Table 3). For each primer combination, an average of 26 fragments was scored and 16 were polymorphic. The highest level of polymorphism $(86.8 \%)$ was obtained with 
Table 4. Total fragments, number of polymorphic fragments and percentage of polymorphic fragment in each primer combination of marker systems.

\begin{tabular}{|c|c|c|c|c|c|}
\hline Primer combination & Total fragments & Polymorphic fragments (\%) & Primer combination & Total fragments & Polymorphic fragments (\%) \\
\hline \multicolumn{6}{|l|}{ TE-TRAP } \\
\hline Sb PIF 1a + odd15 & 42 & $23(54.8)$ & Sb PIF $3+$ ga5 & 48 & $31(64.6)$ \\
\hline Sb PIF $1 \mathrm{a}+$ odd 26 & 38 & $22(57.9)$ & Sb PIF $3+\mathrm{sa} 4$ & 40 & $33(82.5)$ \\
\hline Sb_PIF_1a+ga5 & 37 & $27(73.0)$ & Sb_PIF_3+sa12 & 41 & $35(85.4)$ \\
\hline $\mathrm{Sb}$ PIF $1 \mathrm{a}+\mathrm{sa} 4$ & 40 & $34(85.0)$ & Sb PIF $4+$ odd 15 & 29 & $19(65.5)$ \\
\hline Sb PIF $1 a+s a 12$ & 27 & $17(63.0)$ & Sb PIF $4+$ odd 26 & 29 & $21(72.4)$ \\
\hline Sb_PIF_1b + odd 15 & 40 & $23(57.5)$ & Sb_PIF_4 + ga3 & 43 & $33(76.7)$ \\
\hline Sb_PIF_1b+odd 26 & 38 & $21(55.3)$ & Sb_PIF_4 + ga5 & 42 & $22(52.4)$ \\
\hline Sb PIF $1 b+g a 5$ & 42 & $23(54.8)$ & $\mathrm{Sb}$ PIF $4+\mathrm{sa} 4$ & 45 & $25(55.6)$ \\
\hline Sb_PIF_1b + sa12 & 37 & $22(59.5)$ & Zm_PIF_1 + odd 15 & 31 & $18(58.1)$ \\
\hline Sb PIF $2+$ odd 15 & 40 & $25(62.5)$ & Zm PIF $1+$ odd 26 & 24 & $14(58.3)$ \\
\hline Sb PIF $2+$ odd 26 & 28 & $14(50.0)$ & $\mathrm{Zm}$ PIF $1+\mathrm{ga} 5$ & 25 & $19(76.0)$ \\
\hline Sb_PIF_2 + ga3 & 28 & $21(75.0)$ & Zm_PIF_1 + sa12 & 37 & $16(43.2)$ \\
\hline $\mathrm{Sb}$ PIF $2+$ ga 5 & 39 & $21(53.8)$ & Isaac map + odd 26 & 28 & $17(60.7)$ \\
\hline $\mathrm{Sb}$ PIF $2+$ sa12 & 32 & $21(65.6)$ & Isaac map + ga5 & 28 & $19(67.9)$ \\
\hline Sb_PIF_3 + odd 15 & 48 & $32(66.7)$ & Isaac map + sa12 & 40 & $18(45.0)$ \\
\hline Sb PIF $3+$ ga 3 & 47 & $26(55.3)$ & & & \\
\hline \multicolumn{6}{|l|}{$\overline{\text { AFLP }}$} \\
\hline $\mathrm{AA}+\mathrm{CAA}$ & 31 & $23(74.2)$ & TG + CTA & 20 & $13(65.0)$ \\
\hline $\mathrm{AA}+\mathrm{CAC}$ & 38 & $33(86.8)$ & $\mathrm{TG}+\mathrm{CTC}$ & 18 & $9(50.0)$ \\
\hline $\mathrm{AA}+\mathrm{CAG}$ & 31 & $16(51.6)$ & $\mathrm{TG}+\mathrm{CTT}$ & 19 & $13(68.4)$ \\
\hline $\mathrm{AA}+\mathrm{CAT}$ & 28 & $16(57.1)$ & TT + CAA & 19 & $7(36.8)$ \\
\hline $\mathrm{AA}+\mathrm{CTA}$ & 22 & $14(63.6)$ & $\mathrm{TT}+\mathrm{CAC}$ & 32 & $17(53.1)$ \\
\hline $\mathrm{AA}+\mathrm{CTC}$ & 23 & $15(65.2)$ & $\mathrm{TT}+\mathrm{CAG}$ & 22 & $10(45.5)$ \\
\hline $\mathrm{AA}+\mathrm{CTG}$ & 29 & $23(79.3)$ & $\mathrm{TT}+\mathrm{CAT}$ & 43 & $31(72.1)$ \\
\hline $\mathrm{TG}+\mathrm{CAA}$ & 22 & $13(59.1)$ & TT + CTA & 24 & $16(66.7)$ \\
\hline $\mathrm{TG}+\mathrm{CAC}$ & 27 & $15(55.6)$ & $\mathrm{TT}+\mathrm{CTC}$ & 28 & $11(39.3)$ \\
\hline $\mathrm{TG}+\mathrm{CAG}$ & 29 & $10(34.5)$ & TT + CTG & 30 & $11(36.7)$ \\
\hline $\mathrm{TG}+\mathrm{CAT}$ & 20 & $13(65.0)$ & & & \\
\hline \multicolumn{6}{|l|}{ TD } \\
\hline Sb_PIF_1b + CAC & 10 & $6(60.0)$ & Sb_PIF_2+GTA & 10 & $6(60.0)$ \\
\hline Sb PIF $1 b+C A G$ & 11 & $8(72.7)$ & $\mathrm{Sb}$ PIF $3+\mathrm{GA}$ & 22 & $13(59.1)$ \\
\hline Sb_PIF_1b+CTA & 9 & $5(55.6)$ & Sb_PIF_3+GAA & 11 & $5(45.5)$ \\
\hline Sb PIF $1 b+$ GAG & 7 & $3(42.9)$ & Sb PIF $3+$ GAT & 26 & $12(46.2)$ \\
\hline Sb_PIF_1b+TGC & 6 & $2(33.3)$ & Isaac map + GA & 11 & $4(36.4)$ \\
\hline Sb_PIF_2 + GAA & 19 & $11(57.9)$ & Isaac map + GAA & 15 & $5(33.3)$ \\
\hline Sb_PIF_2+GAC & 16 & $11(68.8)$ & Isaac map + GAG & 22 & $10(45.5)$ \\
\hline Sb_PIF 2 + GCT & 12 & $7(58.3)$ & Isaac map + GCT & 16 & $6(37.5)$ \\
\hline
\end{tabular}

primer combination $\mathrm{AA}+\mathrm{CAC}$, and the lowest level $(34.5 \%)$ was obtained with primer combination TG $+\mathrm{CAG}$ (Table 4). The GD and PIC values ranged from $0.085(\mathrm{TT}+\mathrm{CTC})$ to $0.256(\mathrm{TG}+\mathrm{CTT})$ and from $0.073(\mathrm{TT}+\mathrm{CTC})$ to $0.207(\mathrm{AA}+\mathrm{CTG})$, respectively.

\section{Genetic differentiation}

Based on Nei's genetic distance (Nei, 1972), a phylogenetic tree was constructed based on genetic difference among 21 individuals. Based on genetic difference, the TE-TRAP, TD, and AFLP marker systems produced three, three, and two major groups, respectively. The PCA provided an alternative view of the genetic difference among the 21 individuals. The three groups produced by the TE-TRAP tree clustered within a genetic distance of 0.11 . Group 1 comprised two individuals of $200 \mathrm{~Gy}$ and one individual of $300 \mathrm{~Gy}$, whereas group 2 included two individuals of $200 \mathrm{~Gy}$, three individuals of $300 \mathrm{~Gy}$, and three individuals of $400 \mathrm{~Gy}$. The third group contained one control individual, four individuals of $100 \mathrm{~Gy}$, two individuals of $300 \mathrm{~Gy}$, and two individuals of $400 \mathrm{~Gy}$. The phylogenetic analysis corresponded very well with the PCA for the TE-TRAP marker system (Figures 2 and 3). In contrast, the phylogenetic tree and PCA results based on the TD and AFLP marker systems did not agree, due to unstable groupings produced by the PCA (data not shown).

Genetics and Molecular Research 15 (3): gmr.15038713 


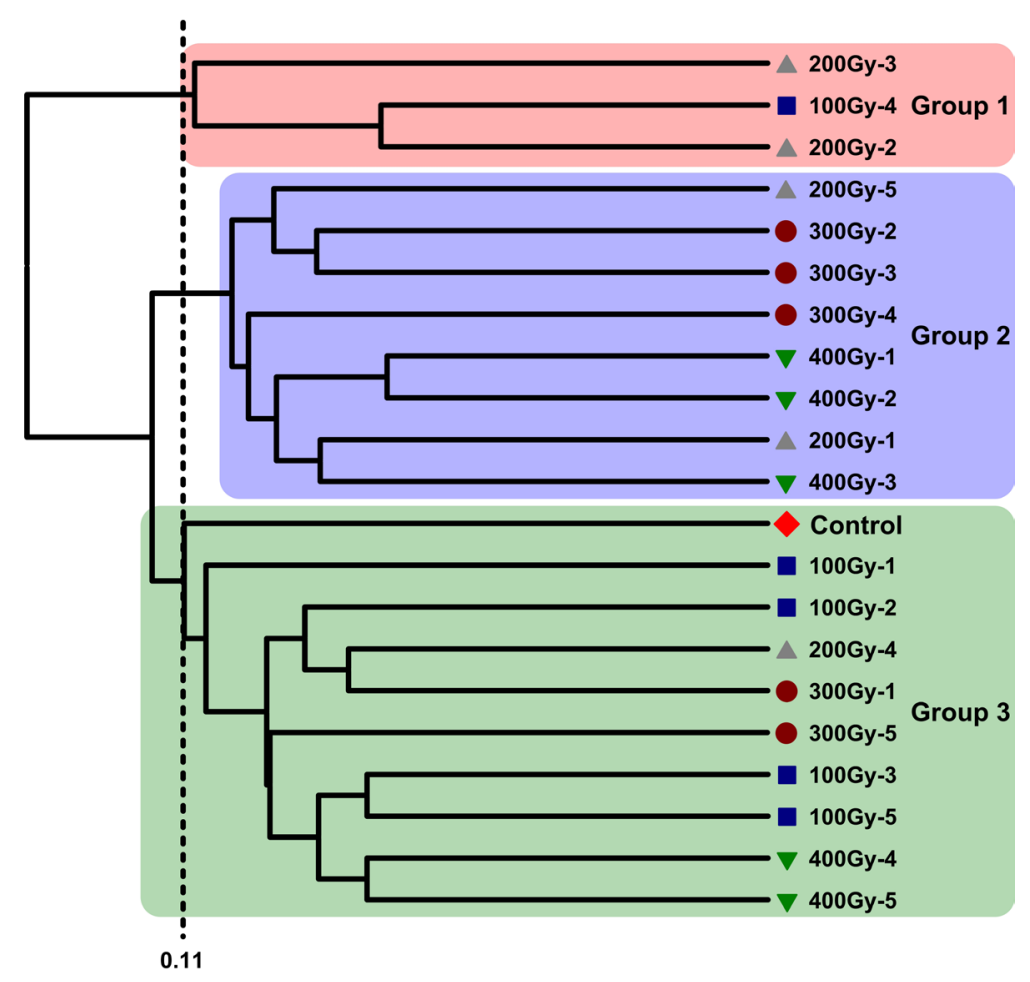

Figure 2. Phylogenetic tree of $21 \gamma$-ray irradiated sorghum (Sorghum bicolor) plants using a TE-TRAP marker system. The dashed line indicates genetic distance at 0.11 .

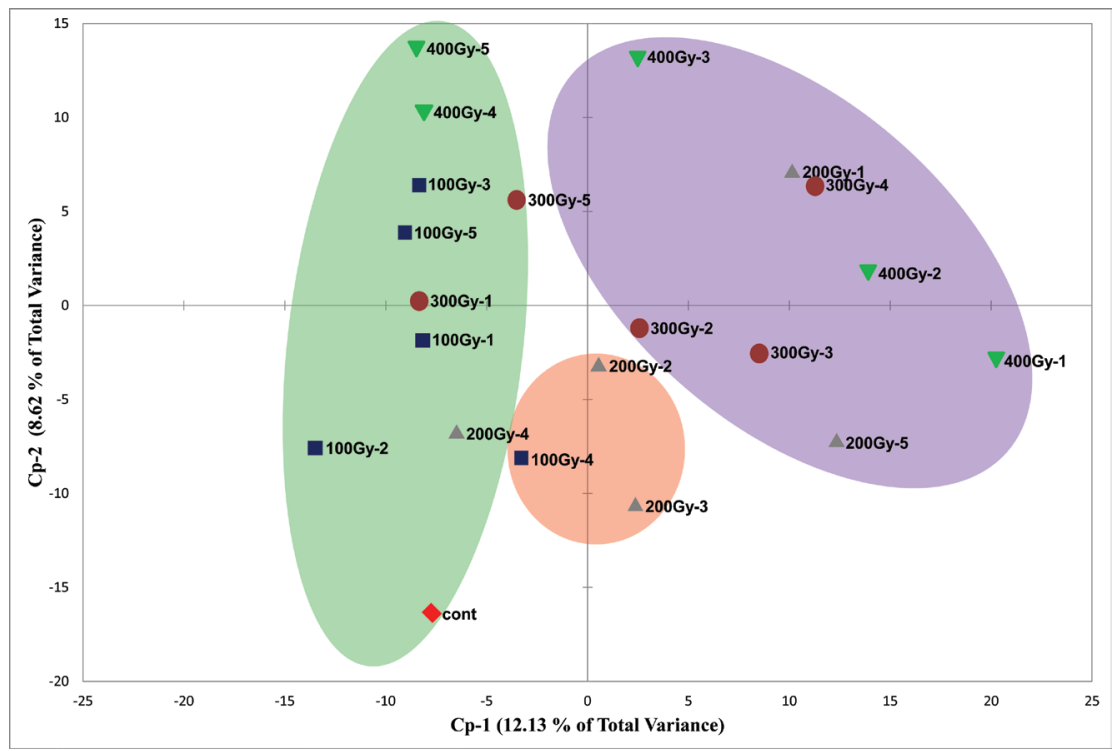

Figure 3. A two-dimensional principal component analysis of $21 \gamma$-ray irradiated sorghum (Sorghum bicolor) plants using TE-TRAP marker system.

Genetics and Molecular Research 15 (3): gmr.15038713 
The genetic distance, differentiation, and AMOVA results were estimated to support the results of the phylogenetic tree and PCA for each marker system (Table 5). The genetic distance ranges of TE-TRAP, TD, and AFLP were 0.211-0.261, 0.137-0.173, and 0.124-0.209, respectively. The highest genetic distance and differentiation between the different groups were observed using the TE-TRAP marker system, whereas the lowest distance and differentiation was observed with the TD marker system. AMOVA conducted among and within each of the four treatment groups (for all five duplications of each dose) revealed that most of the molecular variation in the TE-TRAP marker system explained the most total variation with the among population estimated variance 21.48 and the within population estimated variance 212.61 (Table 6).

Table 5. Analysis of pairwise $F_{\mathrm{ST}}$ values and genetic distances from three different marker systems based on 21 r-ray irradiated sorghum (Sorghum bicolor) individuals.

\begin{tabular}{l|c|c}
\hline & Estimated variance & Percent variation \\
\hline TE-TRAP & & 9.2 \\
\hline Among treatment & 21.48 & 90.8 \\
\hline Within treatment & 212.61 & 100.0 \\
\hline Total & 234.09 & 7.9 \\
\hline TD & 1.77 & 92.1 \\
\hline Among treatment & 20.67 & 100.0 \\
\hline Within treatment & 22.44 & \\
\hline Total & & 10.2 \\
\hline AFLP & 5.32 & 89.8 \\
\hline Among treatment & 46.74 & 100.0 \\
\hline Within treatment & 52.06 & \\
\hline Total & & \\
\hline
\end{tabular}

Genetic distances are shown above diagonal. $F_{\mathrm{ST}}$ values below diagonal. Negative $F_{\mathrm{ST}}$ values were converted to zero.

Table 6. Analysis of molecular variance (AMOVA) from three different marker systems on $21 \gamma$-ray irradiated sorghum plants.

\begin{tabular}{l|c|c}
\hline & Estimated variance & Percent variation \\
\hline TE-TRAP & & 9.2 \\
\hline Among treatment & 21.48 & 90.8 \\
\hline Within treatment & 212.61 & 100.0 \\
\hline Total & 234.09 & 7.9 \\
\hline TD & & 92.1 \\
\hline Among treatment & 1.77 & 100.0 \\
\hline Within treatment & 20.67 & \\
\hline Total & 22.44 & 10.2 \\
\hline AFLP & & 89.8 \\
\hline Among treatment & 5.32 & 100.0 \\
\hline Within treatment & 46.74 & \\
\hline Total & 52.06 & \\
\hline
\end{tabular}

\section{Association analysis between markers and doses}

To study the association between the different markers and the $\gamma$-ray doses, 189 individuals (nine accessions with five individuals of each $\gamma$-ray dose and each original cultivars), which were bred after $\gamma$-ray irradiation, were tested using the TE-TRAP marker 
system. The TE-TRAP marker system generated 1452 markers in the whole population (Figure 4 ) that were scored using a binary code (1 or 0 for presence or absence, respectively).

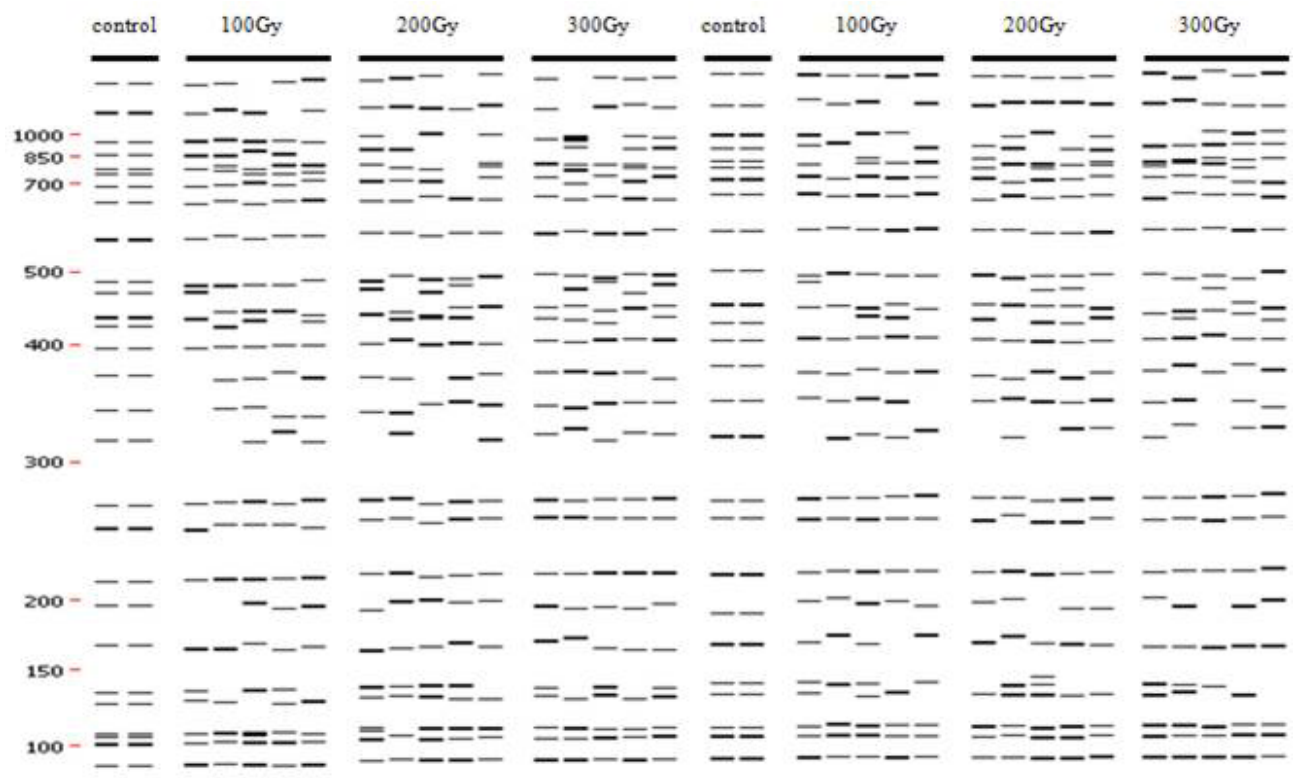

Figure 4. Resulting PCR product pattern of TE-TRAP system with Sb_PIF_2 + Sa4 primer combination for DINEA-MITE cultivar. Two controls were used for validation of ample reproducibility.

The associations between the markers and $\gamma$-ray dose were explored using GLM and MLM $+\mathrm{K}$ methods. Two significant $(\mathrm{P} \leq 0.005)$ associations between the marker and the irradiation dose were identified based on the average of the GLM and MLM + K methods (Table 7). The two markers were Sb_PIF_1a + sa4_15 and Sb_PIF_4 + sa4_35.

Table 7. Results of association analyses between 1452 TE-TRAP markers and different $\gamma$-ray doses (Control, 100, 200, and $300 \mathrm{~Gy}$ ), using two statistical approaches (GLM and K+MLM).

\begin{tabular}{l|c|c|c|c|c}
\hline \multirow{2}{*}{ TE-TRAP marker } & \multicolumn{2}{|c|}{ GLM } & \multicolumn{2}{|c|}{$\mathrm{K}+\mathrm{MLM}$} & \multirow{2}{*}{ Average P } \\
\cline { 2 - 5 } & $\mathrm{R}^{2}(\%)$ & $\mathrm{P}$ & $\mathrm{R}^{2}(\%)$ & $\mathrm{P}$ & \\
\hline Sb PIF 1a+ sa4 15 & 17.2 & $* * *$ & 14.6 & $* * *$ & $* * *$ \\
\hline Sb_PIF_4 + sa4_35 & 12.4 & $* * *$ & 12 & $* *$ & $* * *$ \\
\hline
\end{tabular}

$* * * \mathrm{P} \leq 0.005, * * \mathrm{P} \leq 0.01$.

\section{DISCUSSION}

Sorghum is an important and promising global cereal crop. However, research on sorghum has fallen behind other cereal crops in terms of utilizing molecular biology tools to unwind the complexity of its genome and improve genetic capacity. The use of mutation breeding techniques provides a promising future for sorghum crop improvement. Accordingly, transposon-based marker systems have been developed to select for mutations. 
Conventional TRAP marker arrays are a relatively new PCR-based marker technique (Hu and Vick, 2003). Application of the TRAP marker system to lettuce (Hu et al., 2005), sugarcane (Alwala et al., 2006), sunflowers (Yue et al., 2009), faba beans (Kwon et al., 2010), and chickpeas (Kumar et al., 2014) has suggested that TRAP is suitable for assessing genetic diversity, polymorphisms, and genetic relationships. TRAP uses a fixed primer designed to target expressed sequence tag sequences of candidate genes involved in controlling phenotypes, and amplifies fragments associated with a phenotype. This has been documented by Miklas et al. (2006) for a disease resistance trait in common beans, by Alwala et al. (2006) for sugar content in sugarcane, and by Yue et al. (2010) for the ray flower color in sunflowers.

In this study, TE-TRAP, TD, and AFLP marker systems were applied in 21 individuals to compare the genetic diversity, polymorphism, and genetic differentiation to confirm the efficiency and value of TE-TRAP. For the profile of each marker system, we identified that the TE-TRAP marker system generated more fragments than the other marker systems. TD amplified 223 fragments (avg. 14 amplicons), AFLP amplified 555 fragments (avg. 26 amplicons), and TE-TRAP generated a total of 1133 fragments (avg. 37 amplicons) per primer combination. The percent polymorphic fragments in the TD and AFLP marker systems were 50.8 and $58.4 \%$, respectively, whereas it reached $63.0 \%$ in the TE-TRAP system. As shown in Table 4, the percent polymorphic amplicons per primer combination was large, ranging from 43.2 (Zm_PIF_1 + sa12) to $85.4 \%$ (Sb_PIF_3 + sa12) in the TE-TRAP marker system. The average PIC value of the TE-TRAP markers $(0.172)$ was higher than for TD $(0.122)$ or AFLP (0.132). The GD values for the TD and AFLP marker systems were 0.148 and 0.159 , respectively, whereas the value for the TE-TRAP marker system was higher at 0.214 . Hence, we confirmed the efficiency and applicability of the TE-TRAP marker system, which produced more polymorphic fragments and higher diversity. These results also show that the TE-TRAP marker system is applicable to $\gamma$-ray irradiated sorghum fingerprinting and that the system reveals polymorphisms with high efficiency. Although the TD and AFLP marker systems have high-throughput, both are more labor-intensive and less cost-efficient methods. Therefore, TE-TRAP is an attractive alternative that is easy to perform (not requiring endonuclease treatment), low-cost, and has the high-throughput associated with the use of $\gamma$-ray irradiation.

Notably, we compared the results of a conventional TRAP system and the TE-TRAP system developed in this study, to confirm differences between natural diversity and individual mutant diversity. One previously published study using a TRAP marker system on faba beans (Kwon et al., 2010) estimated the diversity value of faba entries as 0.091 , whereas the diversity based on the TE-TRAP marker system for $\gamma$-ray irradiated sorghum was estimated at 0.214 . These results suggest that mutation breeding increases genetic diversity, although it may have positive or negative impacts. In any event, the TE-TRAP marker system is suitable for identifying the level of diversity in mutation breeding plants.

In this study, the clustering patterns of the phylogenetic tree and the PCA based on genetic distance indicated a distinctive grouping of genotypes based on the TE-TRAP marker system. Three main groups were identified using the TE-TRAP marker system. However, the groups did not appear to be formed based on the $\gamma$-ray doses. It has been shown that physical mutageneses, such as ion-beam, $\mathrm{x}$-ray, $\gamma$-ray, sometimes cause random variance (Kurowska et al., 2012; Kang et al., 2013). In contrast to the TE-TRAP, the phylogenetic tree and PCA based on the TD or AFLP systems did not result in any suitable groupings. We therefore suggest that the TE-TRAP marker system developed in this study allows for more accurate interpretation of the relationship between and among $\gamma$-ray irradiated sorghum individuals than the other 
marker systems. In addition, our results indicate that TE-TRAP markers are useful for differentiating between irradiated sorghum individuals at each of the various $\gamma$-ray doses. The TE-TRAP array was the most sensitive and the most distinguishable marker system for $\gamma$-ray irradiation among the three marker systems. Tables 5 and 6 show the genetic distances and AMOVA results that were supported by grouping the data. The genetic distance and estimated variance of the TE-TRAP marker system were higher than those of the TD and AFLP systems. The highest genetic differentiation found in the TE-TRAP array supported our results that this system could identify the maximum genetic diversity among the marker systems and was the best marker system for $\gamma$-irradiated material.

In Table 5, negative $F_{\mathrm{ST}}$ values were converted to zero. These negative $F_{\mathrm{ST}}$ values indicate that individuals from different doses are genetically more closely related than individuals that received the same dose. As mentioned above, mutations induced by $\gamma$-rays appear to be random with respect to irradiation dose. By genotyping $189 \gamma$-ray irradiated sorghum whole $\mathrm{M}_{1}$ individuals with a TE-TRAP marker array, we conducted an analysis of associations between markers and the $\gamma$-ray doses. We found that the $\gamma$-ray dose was significantly associated with two markers $(\mathrm{P} \leq 0.005)$ : Sb_PIF_1a + sa4_15 and Sb_PIF_4 + sa4_35. This result suggests that TE-TRAP may be used for the analysis of $\gamma$-ray irradiated sorghum and also as candidate genes to select initial mutations.

To date, the genomes of more than 90 plant species have been sequenced, these include 35 food crops. The number of whole plant genomes that are being sequenced is increasing exponentially and our TE-TRAP marker technique can be applied to more plant species to help select initial group mutations. This marker system is likely to be very useful for mutation breeding.

\section{Conflicts of interest}

The authors declare no conflict of interest.

\section{ACKNOWLEDGMENTS}

Research supported by grants from the Nuclear R\&D Program by the Ministry of Science, ICT and Future Planning (MSIP: \#2012M2A2A6003), and the research program of KAERI, Republic of Korea.

\section{REFERENCES}

Alwala S, Suman A, Arro JA, Veremis JC, et al. (2006). Target region amplification polymorphism (TRAP) for assessing genetic diversity in sugarcane germplasm collections. Crop Sci. 46: 448-455. http://dx.doi.org/10.2135/ cropsci2005.0274

Bennetzen JL, SanMiguel P, Chen M, Tikhonov A, et al. (1998). Grass genomes. Proc. Natl. Acad. Sci. USA 95: 19751978. http://dx.doi.org/10.1073/pnas.95.5.1975

Bradbury PJ, Zhang Z, Kroon DE, Casstevens TM, et al. (2007). TASSEL: software for association mapping of complex traits in diverse samples. Bioinformatics 23: 2633-2635. http://dx.doi.org/10.1093/bioinformatics/btm308

Casa AM, Brouwer C, Nagel A, Wang L, et al. (2000). The MITE family heartbreaker (Hbr): molecular markers in maize. Proc. Natl. Acad. Sci. USA 97: 10083-10089. http://dx.doi.org/10.1073/pnas.97.18.10083

Doggett H (1988). Sorghum. 2nd ed. Longman, London; Published in the United states, Wiley, New York.

Doyle JJ and Doyle JL (1987). A rapid DNA isolation procedure for small quantities of fresh leaf tissue. Phytochem. Bull. 19: 11-15.

Genetics and Molecular Research 15 (3): gmr.15038713 
Feschotte C and Pritham EJ (2007). DNA transposons and the evolution of eukaryotic genomes. Annu. Rev. Genet. 41: 331-368. http://dx.doi.org/10.1146/annurev.genet.40.110405.090448

Feschotte C, Jiang N and Wessler SR (2002). Plant transposable elements: where genetics meets genomics. Nat. Rev. Genet. 3: 329-341. http://dx.doi.org/10.1038/nrg793

Hara Y, Abe T, Sakamoto K, Miyazawa Y, et al. (2003). Effects of heavy-ion beam irradiation in rose (Rosa hybrid cv. 'Bridal Fantasy'). RIKEN Accel. Prog. Rep. 36: 135.

Hu J and Vick BA (2003). Target region amplification polymorphism: a novel marker technique for plant genotyping. Plant Mol. Biol. Report. 21: 289-294. http://dx.doi.org/10.1007/BF02772804

Hu J, Ochoa OE, Truco MJ and Vick BA (2005). Application of the TRAP technique to lettuce (Lactuca sativa L.) genotyping. Euphytica 144: 225-235. http://dx.doi.org/10.1007/s10681-005-6431-1

Kang EJ, Lee YM, Sung SY, Ha BK, et al. (2013). Analysis of the genetic relationship of gamma-irradiated in vitro mutants derived from standard-type Chrysanthemum cv. Migok. Hortic. Environ. Biotechnol. 54: 76-81. http:// dx.doi.org/10.1007/s13580-013-0124-9

Kikuchi K, Terauchi K, Wada M and Hirano HY (2003). The plant MITE mPing is mobilized in anther culture. Nature 421: 167-170. http://dx.doi.org/10.1038/nature01218

Kumar Y, Kwon SJ, Coyne CJ, Hu J, et al. (2014). Target region amplification polymorphism (TRAP) for assessing genetic diversity and marker-trait associations in chickpea (Cicer arietinum L.) germplasm. Genet. Resour. Crop Evol. 61: 965-977. http://dx.doi.org/10.1007/s10722-014-0089-2

Kunze R, Saedler H and Lönnig W (1997). Plant transposable elements. In: Advances in botanical research (Callow JA, ed.). Academic Press, Massachusetts, 27: 331-470.

Kurowska M, Labocha-Pawłowska A, Gnizda D, Maluszynski M, et al. (2012). Molecular analysis of point mutations in a barley genome exposed to MNU and gamma rays. Mutat. Res. 738-739: 52-70. http://dx.doi.org/10.1016/j. mrfmmm.2012.08.008

Kwon SJ, Hu J and Coyne CJ (2010). Genetic diversity and relationship among faba bean (Vicia faba L.) germplasm entries as revealed by TRAP markers. Plant Genet. Resour. 8: 204-213. http://dx.doi.org/10.1017/S1479262110000201

Lee JK, Kwon SJ, Park KC and Kim NS (2005). Isaac-CACTA transposons: new genetic markers in maize and sorghum. Genome 48: 455-460. http://dx.doi.org/10.1139/g05-013

Li G and Quiros CF (2001). Sequence-related amplified polymorphism (SRAP), a new marker system based on a simple PCR reaction: its application to mapping and gene tagging in Brassica. Theor. Appl. Genet. 103: 455-461. http:// dx.doi.org/10.1007/s001220100570

Lombardi GM, Nunes JA, Parrella RA, Teixeira DH, et al. (2015). Path analysis of agro-industrial traits in sweet sorghum. Genet. Mol. Res. 14: 16392-16402. http://dx.doi.org/10.4238/2015.December.9.8

Liu K and Muse SV (2005). PowerMarker: an integrated analysis environment for genetic marker analysis. Bioinformatics 21: 2128-2129. http://dx.doi.org/10.1093/bioinformatics/bti282

McClintock B (1950). The origin and behavior of mutable loci in maize. Proc. Natl. Acad. Sci. USA 36: 344-355. http:// dx.doi.org/10.1073/pnas.36.6.344

Miklas PN, Hu J, Grünwald NJ and Larsen KM (2006). Potential application of TRAP (targeted region amplified polymorphism) markers for mapping and tagging disease resistance traits in common bean. Crop Sci. 46: 910-916. http://dx.doi.org/10.2135/cropsci2005.08-0242

Mueller UG and Wolfenbarger LL (1999). AFLP genotyping and fingerprinting. Trends Ecol. Evol. 14: 389-394. http:// dx.doi.org/10.1016/S0169-5347(99)01659-6

Nei M (1972). Genetic distance between populations. Am. Nat. 106: 283-292. http://dx.doi.org/10.1086/282771

Paterson AH, Bowers JE, Bruggmann R, Dubchak I, et al. (2009). The Sorghum bicolor genome and the diversification of grasses. Nature 457: 551-556. http://dx.doi.org/10.1038/nature07723

Peakall R and Smouse PE (2006). GENALEX 6: genetic analysis in Excel. Population genetic software for teaching and research. Mol. Ecol. Notes 6: 288-295. http://dx.doi.org/10.1111/j.1471-8286.2005.01155.x

Rooney LW and Waniska RD (2000). Sorghum food and industrial utilization. In: Sorghum: origin, history, technology, and production (Smith CW and Frederiksen RA, eds.). Wiley, New York, 689-729.

Santiago N, Herráiz C, Goñi JR, Messeguer X, et al. (2002). Genome-wide analysis of the Emigrant family of MITEs of Arabidopsis thaliana. Mol. Biol. Evol. 19: 2285-2293. http://dx.doi.org/10.1093/oxfordjournals.molbev.a004052

Schneider S, Roessli D and Excoffier L (2000). Arlequin ver. 2.000. A software for population genetics data analysis. Genetics and Biometry Laboratory, University of Geneva, Switzerland.

Sneath PHA and Sokal RR (1973). Numerical taxonomy: the principles and practice of numerical classification. Freeman press, San Francisco.

Song HS and Kang SY (2003). Application of natural variation and induced mutation in breeding and functional genomics:

Genetics and Molecular Research 15 (3): gmr.15038713 
papers for international symposium; current status and future of plant mutation breeding. Kor. J. Breed. Sci. 35: 24-34. Tamura K, Dudley J, Nei M and Kumar S (2007). MEGA4: molecular evolutionary genetics analysis (MEGA) software version 4.0. Mol. Biol. Evol. 24: 1596-1599. http://dx.doi.org/10.1093/molbev/msm092

Vos P, Hogers R, Bleeker M, Reijans M, et al. (1995). AFLP: a new technique for DNA fingerprinting. Nucleic Acids Res. 23: 4407-4414. http://dx.doi.org/10.1093/nar/23.21.4407

Wessler SR (2006). Transposable elements and the evolution of eukaryotic genomes. Proc. Natl. Acad. Sci. USA 103: 17600-17601. http://dx.doi.org/10.1073/pnas.0607612103

Yang G (2013). MITE Digger, an efficient and accurate algorithm for genome wide discovery of miniature inverted repeat transposable elements. BMC Bioinformatics 14: 186. http://dx.doi.org/10.1186/1471-2105-14-186

Yu SB, Xu WJ, Vijayakumar CH, Ali J, et al. (2003). Molecular diversity and multilocus organization of the parental lines used in the International Rice Molecular Breeding Program. Theor. Appl. Genet. 108: 131-140. http://dx.doi. org $/ 10.1007 / \mathrm{s} 00122-003-1400-3$

Yue B, Cai X, Vick BA and Hu J (2009). Genetic diversity and relationships among 177 public sunflower inbred lines assessed by TRAP markers. Crop Sci. 49: 1242-1249. http://dx.doi.org/10.2135/cropsci2008.08.0471

Yue B, Vick BA, Cai X and Hu J (2010). Genetic mapping for the Rf1 (fertility restoration) gene in sunflower (Helianthus annuus L.) by SSR and TRAP markers. Plant Breed. 129: 24-28. http://dx.doi.org/10.1111/j.1439-0523.2009.01661.x

Zhang X and Wessler SR (2004). Genome-wide comparative analysis of the transposable elements in the related species Arabidopsis thaliana and Brassica oleracea. Proc. Natl. Acad. Sci. USA 101: 5589-5594. http://dx.doi.org/10.1073/ pnas.0401243101

Genetics and Molecular Research 15 (3): gmr.15038713 